\title{
OPERATIONS PRESERVING ALL EQUIVALENCE RELATIONS
}

\author{
FRED GALVIN AND ALFRED HORN ${ }^{1}$
}

In the recent book by R.S. Pierce [1, p. 38] the following theorem is given as an exercise. If $f$ is a finitary operation on a set $A$ of cardinal $>2$ and $f$ has the substitution property relative to every equivalence relation on $A$, then either $f$ is a constant or $f$ is a projection (that is, there exists an $i$ such that $f(x)=x(i)$ for all $x$ in the domain of $f$ ). We will determine all operations $f$, finitary or not, with this substitution property.

Definition. $|J|$ denotes the cardinal of $J$. If $\beta$ is a cardinal, $\beta^{+}$ is the next larger cardinal.

Definition. If $R$ is an equivalence relation on $A$ and $x \in A^{\alpha}$, $y \in A^{\alpha}$, then we write $x R y$ whenever $(x(i), y(i)) \in R$ for all $i \in \alpha$. An $\alpha$-ary operation $f$ on $A$ is said to have the substitution property relative to $R$ if $(f(x), f(y)) \in R$ whenever $x R y$.

Definition. If $a \in A$, then $R_{a}$ denotes the equivalence relation on $A$ whose equivalence classes are $\{a\}$ and $A-\{a\}$.

Definition. If $\gamma$ is a cardinal, then a filter $F$ of subsets of $S$ is called $\gamma$-complete if $\bigcap_{j \in J} C_{j} \in F$ whenever $|J|<\gamma$ and $C_{j} \in F$ for all $j \in J$. Every filter is $\omega$-complete.

LemMA. Let $\gamma$ be a cardinal $>3$ and suppose $F$ is a family of subsets of $S$. Then $F$ is a $\gamma$-complete prime filter if and only if the following condition holds:

$\left(^{*}\right)$ Whenever $S=\cup_{j \in J} B_{j},|J|<\gamma$, and the $B_{j}$ are pairwise disjoint, then $B_{j} \in F$ for exactly one $j \in J$.

Proof. If $F$ is a $\gamma$-complete prime filter, then the condition is easily seen to hold. Suppose $\left(^{*}\right)$ holds. For any $C \in S$, exactly one of $C$ and $S-C$ is in $F$. Suppose $C \in F$ and $C \subseteq D \subseteq S$. Since $S=C \cup(D-C)$ $\cup(S-D)$, we have $S-D \notin F$. Therefore $D \in F$, and we have shown $F$ is ancestral. Now suppose $C_{j} \in F$ for all $j \in J$ and $J$ is a well ordered set of cardinal $<\gamma$. If $\gamma$ is finite we may assume $|J|=2$ and so in all

Presented to the Society, April 21, 1969 under the title Functions having the substitution property; received by the editors May 30, 1969 and, in revised form, July 14, 1969.

1 The preparation of this paper was supported in part by National Science Foundation Grants GP-8827 and GP-9044. 
cases, $|J|+1<\gamma$. Let $D_{j}=S-C_{j}$ and $D=\cup_{j \in J} D_{j}$. Let $B_{j}=D_{j}$ $-\cup_{i<j} D_{i}$. Then $B_{j} \notin F$ for all $j \in J, D=\bigcup_{j \in J} B_{j}$, and the $B_{j}$ are pairwise disjoint. Since $S=(S-D) \cup \cup_{j \in J} B_{j}$, it follows from $\left(^{*}\right)$ that $S-D \in F$. But $S-D=\bigcap_{j \in J} C_{j}$, and so the proof is complete.

THEOREM. Let $A$ be a set of cardinal $\beta>2$, and let $f$ be a nonconstant $\alpha$-ary operation on $A$, where $\alpha$ is an ordinal. Then the following are equivalent.

(i) $f$ has the substitution property relative to every equivalence relation on $A$;

(ii) $f$ has the substitution property relative to $R_{a}$, for all $a \in A$;

(iii) there exists a $\beta^{+}$-complete prime filter $F$ of subsets of $\alpha$ such that for all $x \in A^{\alpha}, f(x)=$ the a such that $\{i: x(i)=a\} \in F$.

REMARK. If $\alpha$ is finite, or if $\beta$ is infinite and $\alpha$ is less than the first measurable cardinal, the filter $F$ must be principal and so (iii) is equivalent to the assertion that $f$ is a projection.

Proof. It is obvious that (i) implies (ii). The proof that (ii) implies (iii) is divided into parts. Assume $f$ satisfies the hypothesis of (ii). We may assume that $A$ is identified with $\beta$. We first show

(1) For all $x \in A^{\alpha}, f(x)=x(i)$ for some $i$.

Suppose for some $y, f(y)=a$ and $a \neq y(i)$ for all $i$. We show $f$ must be constant. Suppose $f(x)=b \neq a$ for some $x$. There exists $c \in A$ such that $c \neq a$ and $c \neq b$. Let $z \in A^{\alpha}$ be defined by

$$
\begin{aligned}
z(i) & =x(i) & & \text { if } x(i) \neq a, \\
& =c & & \text { otherwise. }
\end{aligned}
$$

Then $z R_{b} x$ and therefore $f(z)=b$. But $z R_{a} y$, since $z(i) \neq a$ for all $i$. Hence $(f(z), f(y))=(b, a) \in R_{a}$, which is a contradiction.

If $B$ is a subset of $\alpha$, we define the sequence $x_{B} \in A^{\alpha}$ by

$$
\begin{aligned}
x_{B}(i) & =0 & & \text { for } i \in B, \\
& =1 & & \text { otherwise. }
\end{aligned}
$$

Let $F$ be the set of all subsets $B$ of $\alpha$ such that $f\left(x_{B}\right)=0$. We show

(2) For any $a \in A$ and any $x \in A^{\alpha}$, we have $f(x)=a$ if and only if $\{i: x(i)=a\} \in F$.

Let $B=\{i: x(i)=a\}$. There exists $b \in A$ such that $b \neq a$ and $b \neq 0$. Let $y(i)=a$ for $i \in B$, and $y(i)=b$ otherwise. Then $x R_{a} y$, and so $f(x)=a$ if and only if $f(y)=a$. Let $z(i)=0$ for $i \in B$, and $z(i)=b$ otherwise. Then $y R_{b} z$, and therefore $f(y) \neq b$ if and only if $f(z) \neq b$. By (1), it follows that $f(y)=a$ if and only if $f(z)=0$. But $x R_{0} x_{B}$, and so $f(z)=0$ if and only if $f\left(x_{B}\right)=0$. Hence $f(x)=a$ if and only if $B \in F$. 
Now we are ready to prove (iii). By (1) and (2), it is clear that $\varnothing \notin F$. Suppose the family $\left\langle B_{j}: j \in J\right\rangle$ satisfies the hypothesis of $\left(^{*}\right)$ in the Lemma, where $S=\alpha$ and $\gamma=\beta^{+}$. By adding enough copies of the empty set we may assume $J=\beta$. Let $x \in A^{\alpha}$ be defined by $x(i)=j$ when $i \in B_{j}$. Then by (2), $B_{j} \in F$ if and only' if $j=f(x)$. Therefore the condition $\left(^{*}\right)$ of the Lemma holds and $F$ is a $\beta^{+}$-complete prime filter. By (2) we see that (iii) holds.

To show that (iii) implies (i), let $R$ be any equivalence relation on $A$. Suppose $x \in A^{\alpha}, y \in A^{\alpha}$ and $x R y$. Then since $\{i: x(i)=f(x)\}$ and $\{i: y(i)=f(y)\}$ are in $F$, there exists $i \in \alpha$ such that $x(i)=f(x)$ and $y(i)=f(y)$. Therefore $(f(x), f(y))=(x(i), y(i)) \in R$, and the proof is complete.

\section{REFERENCE}

1. R. S. Pierce, Introduction to the theory of abstı act algebras, Holt, Rinehart and Winston, New York, 1968. MR 37 \#2655.

University of California, Los Angeles 